Culture et histoire dans l'espace roman

$6 \mid 2011$

Figures du pouvoir dans la littérature hispano-

américaine

\title{
La torture, conceptions du pouvoir chez Carlos Liscano
}

\section{Silvina Benevent Gonzalez}

\section{(2) OpenEdition}

\section{Journals}

Édition électronique

URL : https://journals.openedition.org/cher/9230

DOI : $10.4000 /$ cher.9230

ISSN : 2803-5992

Éditeur

Presses universitaires de Strasbourg

Édition imprimée

Date de publication : 30 juin 2011

Pagination : 291-301

ISBN : 978-2-35410-033-9

ISSN : 1968-035X

Référence électronique

Silvina Benevent Gonzalez, « La torture, conceptions du pouvoir chez Carlos Liscano », reCHERches [En ligne], 6 | 2011, mis en ligne le 17 décembre 2021, consulté le 27 janvier 2022. URL : http:// journals.openedition.org/cher/9230 ; DOI : https://doi.org/10.4000/cher.9230

\section{(c) (i) (2)(2)}

Ce(tte) œuvre est mise à disposition selon les termes de la Licence Creative Commons Attribution -

Pas d'Utilisation Commerciale - Partage dans les Mêmes Conditions 4.0 International. 


\title{
La torture, conceptions du pouvoir chez Carlos Liscano
}

\author{
Silvina Benevent Gonzalez \\ Université Paul Valéry - Montpellier 3
}

La mera existencia es ya resistencia, Le da al más débil un poder que el vencedor no tiene.

Carlos Liscano

\begin{abstract}
T a première caractéristique de Carlos Liscano est d'être devenu écrivain Ldans les geôles uruguayennes. Le pouvoir et ses avatars sont omniprésents dans le titre même de ses œuvres, véritable paratexte par cette inévitable contextualisation. Au-delà des menaces de solitude et de folie, les recueils de nouvelles traduisent une volonté de parler et de transmettre la parole de ceux qui ne peuvent pas parler; l'auteur revendique alors sa fonction de passeur de l'histoire. Parler et écrire. Dans cette mise en rapport du monde du texte et du monde de l'auteur, l'enjeu est un acte de résistance contre un régime qui musèle par la tyrannie et la peur. Et tel est le sens à donner à $E l$ furgón de los locos, pierre angulaire de l'affrontement au pouvoir et à ses représentants.
\end{abstract}

\section{Une nouvelle logique des affrontements}

Ce qui pourrait n'être qu'une entrée en fiction dans El furgón de los locos est brutalement investi d'une puissance terrible par l'intromission d'une épigraphe en italique:

Acaban de traerme de la sala de tortura, que está en la planta baja, bajando la escalera, doblando a la izquierda. Se oyen los gritos, un torturado, otro, y

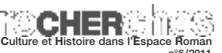


otro, toda la noche. No pienso en nada. O pienso en mi cuerpo. No lo pienso: siento mi cuerpo. Está sucio, golpeado, cansado, huele mal, tiene sueño, hambre. En este momento en el mundo somos mi cuerpo y yo. No me lo digo así, pero lo sé: no hay nadie más que nosotros dos. Pasarán muchos años, casi treinta, antes de que pueda decirme qué es lo que siento. No decirme "qué se siente sino qué sentimos él y yo (Liscano 2001: 7).

Cet incipit provoque, comme le livre, un choc à points d'impact multiples, liés à la brutalité décrite et à la réflexion de l'auteur soumis à cette violence. Le fameux pacte s'instaure par une invitation du lecteur à franchir un seuil quand «decirme» revient à dire aussi à l'autre. Ensuite, la première phrase, apparemment d'une simplicité déconcertante, "Acabo de cumplir siete años » ne manque pas de surprendre le lecteur déjà conditionné par la lecture d'un témoignage poignant. Le narrateur déclenche son récit par la découverte de l'heure, apprentissage oublié par la plupart d'entre nous s'il n'était marqué dans le cas de Liscano par la naissance concomitante de sa petite sœur. Et le lien se fait de façon anodine vers l'arrestation violente, 16 ans plus tard, du jeune Liscano. L'ordre chronologique est certes perturbé mais le narrateur privilégie une organisation intimiste au sein de ce qui se présente comme un triptyque dont le panneau central serait la deuxième partie Uno y el cuerpo, encadrée par Dos urnas y un auto et par Sentarse a esperar lo que sea. Le passé - l’année 1972 est primordiale - devient "hyperprésent”.

La simplicité du préambule ne peut faire oublier l'extrême complexité du propos et manifeste l'annonce de situations-limites. L'espace de la prison ou de la salle de torture est porteur de règles, de valeurs et de rapports nouveaux et insolites: "Era una cárcel rara, una especie de reino negativo del logos» (Liscano 2000b: 25). Dans une situation normale, chacun cherche dans la rencontre et la communication avec autrui à produire une image de soi acceptable et valorisée. Dans les relations entre bourreaux et torturés, l'enjeu fondamental de l'interaction avec autrui est perverti.

Les tortionnaires sont de ce point de vue évoqués selon leur grade militaire qui a valeur d'identité et d'identifiant. Ces dénominations métonymiques vont de pair avec l'utilisation du pluriel et ce, dès le prologue. Ce pluriel non identifiable désigne sans nommer car, comme le dit Vincent Jouve, «Indicateur d'individualité, le nom propre s'affirme comme un support privilégié de l'effet-personne» (Jouve 1992: 111) ce que l'auteur refuse. Le torturé est seul face à un groupe dans cette représentation déshumanisée du bourreau qui n'est pas sans évoquer un monstre à plusieurs têtes. La 
profonde réflexion que mène Liscano s'ébauche à partir d'un questionnement intellectuel et une curiosité même vis-à-vis du bourreau. Qui est-il ? Qui sont ces hommes capables de mener un semblable aux limites de la souffrance? Plusieurs chapitres sont donc consacrés à cette figure du tortionnaire et à sa responsabilité en tant qu'être humain. Liscano l'examine et fait part des hiérarchies: le torturé est la possession d'un responsable. Au-delà de la description objective du tortionnaire, il y a une mise en lumière de la représentation du tortionnaire par sa victime «mezcla de padre autoritario y castigador, señor de sus esclavos, pequeño dios que administra el dolor, la comida, el agua, el aire, el abrigo, la higiene, las idas al baño" (Liscano 2001: 86) et il n'échappe pas à l'ironie de l'écrivain: «Oficio duro el de torturador, exige fuerza, decisión ¿olvido de sí mismo?» (72).

Afin de mieux cerner les besoins des victimes de tortures, Françoise Sironi s'est intéressée à la fabrication du tortionnaire. Elle a ainsi étudié les processus d'initiation des tortionnaires qui utilisent le même mécanisme qu'à leur tour ils infligeront à leur victime: l'alternance de phases, un ordre binaire (le bon et le méchant), l'organisation délibérée d'un traumatisme de nature intellectuelle, la déconstruction identitaire et une expérience incommunicable (Sironi 1999: 139-141). Désormais, le tortionnaire est totalement convaincu d'être au-dessus de la loi, d'être un homme à part, ce qui explique son incapacité à vivre par la suite dans la nouvelle société. Quant au torturé, toute la complexité du phénomène se révèle dans cette quête/ enquête sur l'autre car il cherche une similitude entre lui-même et son interlocuteur quand il voit son propre sentiment d'humanité entièrement mutilé. Rappelons que la constitution presque mythique du je s'opère depuis une reconnaissance de la mère (ou de la figure maternelle) et un accès simultané à son propre corps par différenciation dans la phase $d u$ miroir décrite par Lacan. Or le miroir est ici brisé, le protagoniste est clairement séparé du reste de l'humanité, «Había que congelarles todo movimiento, acción y pensamiento» (Liscano 2000b: 25). La phase dite inaugurale apparaît marquée dans le prologue "somos mi cuerpo y yo" (Liscano 2001: 7) dans le processus inverse: la perte de conscience de son propre corps. Cette décorporéisation est conséquence de la torture psychologique et traumatisante qui tente de chosifier la victime, de la réduire en tant que personne.

En effet, la première mise en scène du torturé par ses tortionnaires est le ligotage des mains et le geste de la cagoule. Les soldats qui l'arrêtent la mettent immédiatement en place, il y a déjà négation de l'individu et cela 
avant même l'épreuve de la torture. Généralement, en entrant dans un lieu inconnu, nous parcourons des yeux la pièce, l'assemblée pour y trouver des repères et un visage ami, cela est sécurisant. La cagoule de facto brise cet élan et par là même la fonction première du sentiment d'identité qui est d'assurer à l'individu le sens d'une intégration et d'une continuité face à l'inconnu. Il faut observer enfin que l'imposition de la cagoule est le geste qui ôte sa singularité à l'individu en lui cachant le visage dans la mesure où il est ce que chacun a de plus personnel.

Remarquons encore que la torture attaque la part collective de l'individu et cherche à l'isoler de son groupe (F. Sironi parle d'effet de déculturation). De ce point de vue, un épisode est révélateur de ce processus d'influence. Liscano est emmené afin d'identifier un agent dont il ne connaît que le pseudonyme (Francisco). Il attribue tout d'abord son visage livide à la torture avant de comprendre qu'il est mort. Toute la mise en scène qui a valeur d'épreuve (Liscano va-t-il l'identifier?) vise manifestement à délier le lien entre les camarades et à précipiter la destruction psychologique. Tel est le sens enfin des menaces proférées à l'encontre de Liscano, par un capitaine avant de l'interroger:

Me dice que a un compañero, al que yo conozco, él lo tiene en su cuartel y lo ha dejado hecho un animalito.

"Camina a cuatro patas, como un animalito.

Así te voy a dejar a vos" (Liscano 2001: 155).

La torture, instrument de pouvoir, cherche à ébranler l'identité même de l'être humain par des attaques répétées du corps et par l'agression portée au sentiment d'humanité. Elle poursuit la désintégration de la personne par la négation des besoins, des moyens de défense et des liens.

\section{Le corps comme point d'impact du pouvoir}

$\mathrm{Au}$ demeurant, le pouvoir est une perception de soi comme inférieur ou supérieur à autrui selon le point de vue où on se place. Par la torture, l'image, la représentation et l'estime de soi sont non seulement dégradées mais définitivement anéanties. La propre personne telle que se la représente le torturé, est un corps-objet à travers les regards des tortionnaires. Un processus de néantisation apparaît déjà dans l'interpellation du prisonnier (le supplicié est un pseudonyme, le prisonnier, un chiffre) car «La "solución final" [...] era la destrucción mental y física de los presos» (Liscano 2000b: 25). La violence donne à l'un une légitimité d'état qui est niée à l'autre. 
Aussi le choix onomastique dans les œuvres de Liscano est à première vue celui de l'absence. Lorsque l'auteur nomme, il désigne en toute conscience des personnes identifiables par le lecteur. Ainsi, dans La ciudad de todos los vientos, un narrateur incarne un véritable héros qu'il désigne comme $\mathrm{C}$, puis $\mathrm{C}-$, une identité encore tronquée. Le narrateur à la recherche de l'inspiration parcourt Montevideo, et rencontre Liscano lui-même: «¿Tenés idea de por qué escribís? ¿Y por qué escribís como escribís? Yo intenté leer La mansión del tirano, te lo juro" (Liscano 2000a: 23) et plus tard, Benedetti. Ces épisodes métatextuels le sont précisément par l'incrustation de noms réels et reconnaissables. Néanmoins, l'auteur donne la priorité, dans El furgón de los locos, au lien que les tortionnaires ont tenté de briser et nomme ses compagnons d'infortune, ses parents et sa femme. De même, se nommer dès les premières pages est une revendication de son existence en tant que personne "Ya he aprendido a aclarar que no soy Lescano ni Lascano ni Lezcano. Liscano, con i y con s. Una vida explicándolo» (Liscano 2001: 11). De là peut-être, l'obsession de la première personne, et ce je, loin d'évoquer un tic exclusivement narcissique est une revanche face à la menace de trouble psychique majeur, celui de la dépersonnalisation. Si le corps persécuté, morcelé, déchiré et atrophié a fini par se séparer de la tête, comme lieu de pensée et de la parole, il n'est pas anodin de remarquer que le pronom indéfini "uno» est particulièrement présent dans cet ouvrage. Sous couvert du pronom, l'auteur semble se détacher de son récit tout en s'impliquant. Mais ce constant va-et-vient transcrit surtout la dualité de celui qui parle: «Pero uno tiene que soportarse a sí mismo» (101).

La situation limite implique de fait un langage lui aussi à la limite du sens. La parole perd dans l'univers des geôles sa fonction merveilleuse de transmission. Elle a brutalement, pour unique fonction, celle de créer la peur et de provoquer la délation. Le sens de la parole, perverti, est désormais accaparé par l'autre: «La palabra es la cultura, y la cultura, propiedad de los vencedores, es mentira, represión, arbitrariedad, maltrato [...] El preso desconfía del lenguaje $[. .$.$] es peligroso, porque puede llevar al descreimiento$ total en el ser humano, que es la palabra» (Liscano 2000b: 35). L'acte même de parler se double de son antonyme, le silence, car partager sa parole reviendrait à faire acte de collaboration face au pouvoir. Elle serait alors la parole qui délivre mais rend coupable. En revanche, se taire c'est supporter le supplice. Tel est le dilemme induit par la situation de torture que le supplicié doit affronter. La parole sous influence, l'échange instrumentalisé inférent, dans ce cas précis un profond clivage entre le dire et se taire. La 
parole est tour à tour surinvestie et dévalorisée car dévalorisante. Relevons que la bouche, tout en perdant ses fonctions vitales (respirer, manger), concentre entièrement l'enjeu de transmettre l'oral et devient brusquement dangereuse. Dans un univers où le langage est dénaturé, le langage humain redevient unité primaire: "No son gritos normales de dolor, sino como de bestia, de animal desesperado. No le dan la boca y la nariz para conseguir aire. El sonido sale entrecortado, como una sucesión de explosiones. Es un bramido más que un grito» (Liscano 2001: 68).

Corollaire de la parole, l'ouïe fait naître de la même façon des sentiments de persécution par une pénétration incontrôlable d'éléments perturbateurs. L'acuité auditive est à tel point accrue que Liscano reconnaît lors d'une réunion amicale à Stockholm, le cri et le rire d'une ancienne détenue qu'il n'avait jamais vue: "El oído había conservado aquel grito y aquella voz estridente» (49). La promiscuité avec les autres torturés s'exerce exclusivement dans l'audition ${ }^{1}$ puisque les cris sont omniprésents : «Durante la noche se oyen los gritos de hombres, mujeres, el ladrido de los perros que los militares azuzan contra los torturados para amedrentarlos. Los oficiales también gritan, amenazan, insultan» (64).

La torture, dans sa démarche de rupture délibérée avec l'univers de référence, met en activité un autre de nos sens en transgressant les tabous culturels du torturé. Nous pensons à l'odorat, dont on se défend car il touche trop à l'animalité de l'homme. Or l'intensification du sens olfactif va de pair avec l'organe auditif dans El furgón de los locos: "Hay olor a tabaco, a sudor, a alcohol, a orín, a desinfectante de excusado. [...] Aquí hay olor a dos tipos de miseria: la del torturado, y la de los torturadores » (76-77). Cet aspect essentiel dans le récit met en évidence la permanence des prisonniers dans une saleté imposée et produite par le prisonnier lui-même qui est irrémédiablement marqué par la souillure, «La mugre es otra puerta al autoconocimiento. [...] Uno puede decir, como imagen fuerte, "esto da asco". Es distinto sentir: "ahora doy asco"»(101).

Les phénomènes physiques sont d'autant plus vitaux qu'ils ne sont plus si naturels. Obtenir l'autorisation de se soulager est vital et sans pudeur, Liscano décrit l'obsessionnel rituel de l'acte d'uriner et de déféquer. Cela étant, toute matière qui sort du corps du torturé est inévitablement objet de répulsion et d'humiliation. Liscano détaille la méthode du tacho, un

1 Liscano décrit au contraire dans El lenguaje de la soledad les effets dévastateurs de l'isolement total. 
baril coupé en deux rempli d'eau dans lequel les tortionnaires plongent leur victime cagoulée jusqu'au point ultime de l'asphyxie. Ensuite, l'eau retenue par la cagoule se mêle aux cheveux, aux vomissures et à la salive et prolonge le supplice. Excréments, urine, vomissements, transpiration et crasse, tout concourt donc à maintenir l'individu dans une animalité dépravante.

A la négation des besoins les plus simples, répond la nécessité pour le prisonnier d'exister dans une structure spatio-temporelle définie et déterminable. Ainsi, la suppression de la capacité à voir incite-t-elle l'individu à complexifier sa perception de l'espace. Comment ne pas s'étonner de l'importance donnée par Liscano à la représentation spatiale de ses souvenirs? Il sait parfaitement où se trouve la salle de torture - il nous l'indique dès le prologue - ainsi que la caserne où on l'interroge, «fui capaz de seguir mentalmente las calles por donde me llevaban y sé en qué cuartel estamos. Ahora, encapuchado en el piso del camión la cabeza sigue la ruta» (Liscano 2001: 147). Plus tard, il perçoit avec certitude les lieux où on l'emmène et parfois l'indication du lieu débute le chapitre (par exemple, la préfecture de Police de Montevideo). A vrai dire, le détenu a besoin de s'imaginer spatialement dans l'univers carcéral. Cette obsession est ce qui le pousse à construire une description précise et presque scientifique du pénitencier de Libertad dans El lenguaje de la soledad: «El edificio, a unos diez metros sobre el suelo, sostenido por 96 columnas, estaba dividido en cinco pisos, que se dividían en dos sectores, que se dividían en dos alas. Las barracas estaban divididas en dos sectores cada una» (Liscano 2000b: 26).

L'auteur investit cependant son espace sans chercher à créer des effets de réel. Parfaitement décrit, l'espace, vide un temps, ne laisse pas de place à l'imaginaire du lecteur mais se remplit des attentes et des révoltes du prisonnier. La vérité est dite, crue. En effet, l'espace de référence est déterminé par Liscano selon des critères récurrents: la lumière et son absence, une ouverture, les dimensions, le mobilier et la fonction de la pièce. Prenons un exemple, "A los pocos días me trasladan a una celda del sexto piso de Jefatura de Policía. Hay un camastro, no hay colchón, la ventana está tapiada. La celda es tan pequeña que no se puede ni caminar ni estar de pie [...] Poco a poco empiezo a hacerme una idea del lugar» (Liscano 2001: 154). S'orienter et investir le lieu marque en conséquence une implication du je dans un ici et un maintenant qui vise à préserver son identité face au danger immédiat. Il en va de même de la notion temporelle qui, elle aussi, incarne la permanence de l'être. Constatons que Liscano s'applique à garder en mémoire les dates qui sont marquantes. Le temps primordial, c'est celui 
de l'événement mais, dans ce cas précis, il n'est pas fondateur, au contraire, il est destructeur. Le temps et l'espace se conjuguent donc pour sécuriser le moi qui se souvient et ils sont ainsi la preuve d'un passé révolu; ils sont des points d'ancrage entre l'histoire vécue et l'histoire sociale pour échapper à l'anéantissement.

L'expérience sensorielle oscille en l'occurrence, entre deux pôles antagonistes qui finissent par établir un point d'équilibre nécessaire à la survie: «Para salir adelante tengo el instinto del animal en el monte, que es el hábito del preso: ver sin mirar, oír sin escuchar, estar enterado sin demostrarlo» (Liscano 2001: 46). Une parole qui ne dit rien, des yeux qui ne regardent pas sont la marque de l'ensemble des conduites et des mécanismes (cognitifs, affectifs, défensifs) que l'individu met en œuvre dans son entêtement à être.

Mais si l'on considère la réflexion de Liscano sur la parole dite, tue ou arrachée, on remarque qu'elle est l'objet de toutes ses attentions, en tant qu'homme qui manipule la parole écrite. Quand l'homme est vaincu, réprimé, réduit à rien, effacé de l'histoire, il ne lui reste que sa parole: «En ese momento uno descubre lo que siempre supo pero nunca necesitó formularse: que el que es, es por la palabra» (Liscano 2000b: 29). La parole qui s'auto-détruit pour survivre puis s'auto-questionne et s'auto-alimente finit par s'auto-incarner, elle est à nouveau fondatrice.

\section{Le contre-pouvoir ou la voie/voix de la résilience}

Après avoir côtoyé la mort et la détresse humaine, contre toute attente, le supplicié défie le pouvoir par sa seule existence. Une liberté qui, d'un coup, redevient accessible et participe alors d'un banal extraordinaire. Elle sera et reste pour l'écrivain un sujet primordial: «Es el problema más serio del ser humano. Para el preso, la libertad es resistir» (entretien avec l'auteur: 18-10-2010). Néanmoins, face au traumatisme sciemment provoqué, il faut faire acte de libération et elle passe par la résilience. La résilience est «un terme habituellement employé en physique des matériaux pour désigner la "résistance" d'un matériau aux chocs élevés et la capacité pour une structure d'absorber l'énergie cinétique du milieu sans se rompre» (Anaut 2008: 35). Écrire, se raconter, c'est pour Liscano résister à l'agression du régime qui a cherché à l'effacer et ce, sans se rompre. Mais le mécanisme se met déjà en place en prison et passe par la prise de décision: «Aquí me haré hombre adulto [...] Comenzaré a escribir. Decidiré que seré escritor» (Liscano 
2001 : 181). Subrepticement, contre l'atteinte faite à la capacité de penser en prison, l'écriture prend consciemment la forme d'une thérapie. De fait, la pensée comme moyen de supporter l'accroissement des tensions psychiques permet, chemin faisant, d'attribuer un sens à la blessure: "Quería hacer una reflexión sobre la condición humana" nous a-t-il confié à propos de El furgón de los locos (entretien 18-10-2010). Et l'acte d'humanité surgit, lumineux, quand un soldat l'aide à uriner malgré les menottes et malgré la honte (Liscano 2001: 151). Pourtant, dire les choses, c'est les éprouver de nouveau, d'une autre manière. Qui plus est, faire le récit de soi-même, se mettre à nu demande du courage car derrière les mots, tout est affectif. Dès lors, l'enjeu du récit est de taille: se sauver.

L'effet est de la même importance puisqu'un lien d'intimité se tisse avec le lecteur, le fameux pacte est véritablement incarné. Un narrateur-témoin versus un lecteur-auditeur - «Estimada lectora, atento lector", lit-on dans La ciudad de todos los vientos (Liscano 2000a: 67) - remplissent les fonctions essentielles qu'attribue F. Sironi à la thérapie des victimes de la torture: trouver un interlocuteur. Il s'agit toutefois d'écouter une voix agissante: «de golpe, la voz se abrirá camino, se me impondrá, querrá decir, contar, con o sin jerarquía, con o sin calidad literaria. Y la voz se hará indetenible, me dirá qué escribir, rescatará hechos, sensaciones, sentimientos que no recordaba» (Liscano 2001: 183). Cette parole active est cette transgression ultime du prisonnier dont la survie est un véritable pied de nez à l'histoire. En effet, le processus de résistance implique aussi un devoir de survie, véritable contrepouvoir: «El preso, para el guardián, es un misterio. Nunca sabrá quién es. Eso hace del preso un ser poderoso, y libre» (Liscano 2000b : 33).

La narration de la souffrance, outre le fait de permettre la communication avec les autres est un récit vivifiant dans la mesure où il impulse un avenir et met en pratique ce que Boris Cyrulnik nomme «donner de l'avenir à l'avenir » (Cyrulnik 1999: 78), c'est-à-dire se développer de manière positive malgré une situation à haut risque. Dès sa libération, Liscano se place dans l'agir: «Al otro día me levantaré a las cinco y media de la mañana, obsesionado por hacer "algo" con mi libertad [...]. Esa mañana sentiré que la vida me pertenece, que es mía, sólo mía, y que puedo hacer con ella lo que quiera " (Liscano 2001: 182). Cela rejoint la course obsessionnelle du narrateurauteur de La ciudad de todos los vientos: "Ante tanta dificultad, el autor sale a la calle Reconquista a ver si encuentra algo para escribir, y correrá unas doscientas páginas» (Liscano 2000a: 20). Cependant, cette course se déploie selon un axe topographique précis de Montevideo. En s'inventant auteur 
dans la capitale, Liscano se réapproprie sa ville et son entité d'Uruguayen, lui qui a aussi connu l'exil. Cela relève d'ailleurs du contraire de la dispersion (se perdre) dans une reconquête du sentiment de soi. A l'évidence, l'estime de soi, comme critère remarquable de la résilience selon Michael Rutter (Anaut 2008: 49), fait obstacle aux tentatives de déstructuration imposées par la torture. A cet égard, l'extrême capacité à se projeter dans la vie est sous-tendue chez cet auteur par son sens de la dignité: «Lo único que le pedí a mi cuerpo en la tortura fue que me permitiera algún día mirarlos a la cara con dignidad» (Liscano 2001: 185).

Il faut remarquer enfin que la réaction cathartique se produit curieusement grâce à l'humour. Ce dernier fait partie des mécanismes salvateurs de la résilience (Anaut 2008: 90) et n'est pas sans rappeler le défi à rebondir ${ }^{2}$ que le terme résilience porte en lui : «Cuando nada puede estar peor, no hay nada de qué reírse. Pero entonces, como nadie es más grotesco que un preso, todo puede ser motivo de risa. Esto acaba por dar una extraña fuerza: uno pasa a ser blanco de sus propias bromas, y así se salva. El lenguaje vuelve a salvarlo » (Liscano 2000b : 34). Il est ainsi un rempart contre l'anéantissement.

Nous avons pu voir que sous la torture, la sensation de distorsion entre corporel et psychique naît de la négation faite au torturé de son humanité et va jusqu'à ébranler la conscience de soi. Qui plus est, l'agression physique se double d'une conscience d'être victime d'un semblable. Son sentiment d'impuissance est total face à cette injustice de ne pas être responsable de son destin ni de son mal-être.

Du reste, dans une recherche effrénée de la permanence ontologique, des notions antinomiques se juxtaposent plus qu'elles ne s'articulent sous l'effet de la contrainte: se perdre /s'orienter, parler /se taire, entendre /crier, regarder /occulter, oublier/transmettre. Il y a en définitive mise à l'épreuve de l'estime de soi dont Liscano triomphe par un sentiment exacerbé de la dignité humaine, valeur qui a survécu à tous les dangers. Mais le triomphe passe par un jeu subtil de différents facteurs, un maillage selon Cyrulnik ${ }^{3}$ qui a sans doute permis à l'écrivain de dépasser le conflit psychique dérivé de la torture.

Cette reconquête lente et semée d'embûches investit tout entière la parole et fait de l'écrivain le sujet même du langage:

2 Le verbe resilio signifie sauter en arrière, rebondir, rejaillir.

3 Cyrulnik explique que «la résilience est un tricot qui noue une laine développementale avec une laine affective et sociale. [...] c'est un maillage. Nous sommes tous contraints de nous tricoter avec nos rencontres dans nos milieux affectifs et sociaux» $(15,43-44)$. 
El mayor invento de un escritor es la invención de sí mismo como escritor. Tarde o temprano el que escribe debe inventar a ese escritor que aún no existe, inventarle la voz, inventarle la obra que habrá de escribir, renunciar a lo que no habrá de escribir, inventarle un modo de estar en el mundo (Liscano 2000a: 49).

En définitive, au-delà de la haute transmissibilité du témoignage de la torture, il y a une re-prise de possession de sa personne et de son monde digne et libératoire: "Qué me importa la novela. Era solo esto lo que necesitaba, dormirme cara al sol, a esperar el próximo milenio, en la Ciudad de todos los vientos. Y ahora sí, la vida puede comenzar» (52).

\section{Bibliographie}

Anaut Marie, 2008, La Résilience. Surmonter les traumatismes, Paris, Armand Colin.

Cyrulnik Boris, 1999, Un merveilleux malheur, Paris, Odile Jacob. Jouve Vincent, 1992, L'effet-personnage dans le roman, Paris, PUF. Liscano Carlos, 2000a, La ciudad de todos los vientos, Montevideo, Planeta. Liscano Carlos, 2000b, El lenguaje de la soledad, Montevideo, Cal y Canto. Liscano Carlos, 2001, El furgón de los locos, Montevideo, Planeta. Sironi Françoise, 1999, Bourreaux et victimes, Paris, Odile Jacob. 